\title{
Evidence for de novo acquisition of microalgal symbionts by bleached adult corals
}

\author{
Hugo J. Scharfenstein (D) ${ }^{1,2^{凶}}$, Wing Yan Chan ${ }^{1}$, Patrick Buerger ${ }^{1,3}$, Craig Humphrey $\mathbb{D}^{2}$ and Madeleine J. H. van Oppen (iD ${ }^{1,2}$ \\ (c) The Author(s) 2022
}

Early life stages of most coral species acquire microalgal endosymbionts (Symbiodiniaceae) from the environment, but whether exogenous symbiont uptake is possible in the adult life stage is unclear. Deep sequencing of the Symbiodiniaceae ITS2 genetic marker has revealed novel symbionts in adult corals following bleaching; however these strains may have already been present at densities below detection limits. To test whether acquisition of symbionts from the environment occurs, we subjected adult fragments of corals (six species in four families) to a chemical bleaching treatment (menthol and DCMU). The treatment reduced the native microalgal symbiont abundance to below $2 \%$ of their starting densities. The bleached corals were then inoculated with a cultured Cladocopium $\mathrm{C}^{\text {acro }}$ strain. Genotyping of the Symbiodiniaceae communities before bleaching and after reinoculation showed that fragments of all six coral species acquired the Cladocopium $\mathrm{C}^{\text {acro }}$ strain used for inoculation. Our results provide strong evidence for the uptake of Symbiodiniaceae from the environment by adult corals. We also demonstrate the feasibility of chemical bleaching followed by reinoculation to manipulate the Symbiodiniaceae communities of adult corals, providing an innovative approach to establish new symbioses between adult corals and heat-evolved microalgal symbionts, which could prove highly relevant to coral reef restoration efforts.

The ISME Journal (2022) 16:1676-1679; https://doi.org/10.1038/s41396-022-01203-0

Scleractinian corals prosper in oligotrophic waters by forming mutualistic relationships with microalgae (Symbiodiniaceae) that translocate photosynthate to their host [1]. Breakdown of the coral-Symbiodiniaceae symbiosis, i.e. coral bleaching, occurs in response to environmental stress and may result in widespread coral mortality [2]. Thermal stress is the primary cause of largescale coral bleaching events, which have become more frequent and severe over recent years as climate change-driven marine heatwaves gain prevalence [3].

Bleaching tolerance of corals to elevated temperatures varies within and among species. This is partly determined by the physiological performances of their microalgal symbionts under thermal stress $[4,5]$. For instance, thermotolerant Symbiodiniaceae species in the genus Durusdinium have been found to increase the bleaching threshold of the coral holobiont by $1-2{ }^{\circ} \mathrm{C}[6]$.

The Symbiodiniaceae comprise at least 15 genera and genuslevel lineages which include many species [7]. Several microalgal symbionts may coexist within a coral host, with the Symbiodiniaceae communities of adult corals often being dominated by a single species [8]. Other members of the symbiont community are found in low abundances, forming a Symbiodiniaceae rare biosphere increasingly linked to coral bleaching resilience [9]. Changes in the relative abundance of Symbiodiniaceae species (shuffling) [10] and the acquisition of new symbionts from the environment (switching) [11] in adulthood are potential mechanisms for corals to adapt to increases in sea surface temperatures [4-6, 8-11]. Evidence of symbiont shuffling is widespread $[4,5,10]$, yet reports of switching in adult corals remain limited to metabarcoding studies [12] and the Symbiodiniaceae rare biosphere [11], where the acquired symbionts may have been present below the detection limit before bleaching. Whilst environmental uptake of exogenous Symbiodiniaceae has been demonstrated in adult sea anemones [13] and octocorals [14], experimental evidence of switching in adult corals remains unconvincing with only a transient symbiosis being reported in adult colonies of Porites divarcata following bleaching [15].

Here we used chemical bleaching to remove $>98 \%$ of the native symbiont cells from adult fragments of six coral species spanning four families (i.e. Diploastrea heliopora (Diploastraeidae), Dipsastraea pallida (Merulinidae), Echinopora lamellosa (Merulinidae), Platygyra daedalea (Merulinidae), Porites lobata (Poritidae) and Stylophora pistillata (Pocilloporidae)), which were then successfully reinfected with a cultured Symbiodiniaceae strain. Colonies were fragmented and chemically bleached ( $n=16$ per species) through exposure to menthol and 3-(3,4-dichlorophenyl)-1,1-dimethylurea (DCMU; Supplementary methods section 1.3). Once bleached, the coral fragments underwent four different reinoculation treatments ( $n=3$ per species and treatment): (1) a negative control treatment (Ctl-) where corals were not reinoculated with any Symbiodiniaceae; (2) a positive control treatment $(\mathrm{Ctl}+)$ where corals were reinoculated with freshly isolated homologous Symbiodiniaceae (obtained through tissue blasting of conspecific coral fragments); (3) a reinoculation treatment $(\mathrm{Ri})$ where corals were reinoculated with a cultured Cladocopium C1 ${ }^{\text {acro }}$ strain (SCF055-01.10) at $10^{4}$

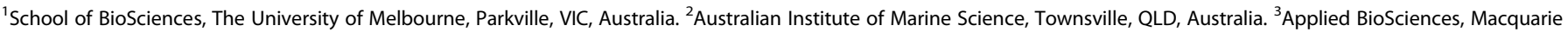
University, Sydney, NSW, Australia. ${ }^{\circledR}$ email: hscharfenste@student.unimelb.edu.au

Received: 12 October 2021 Revised: 24 January 2022 Accepted: 25 January 2022

Published online: 7 February 2022 


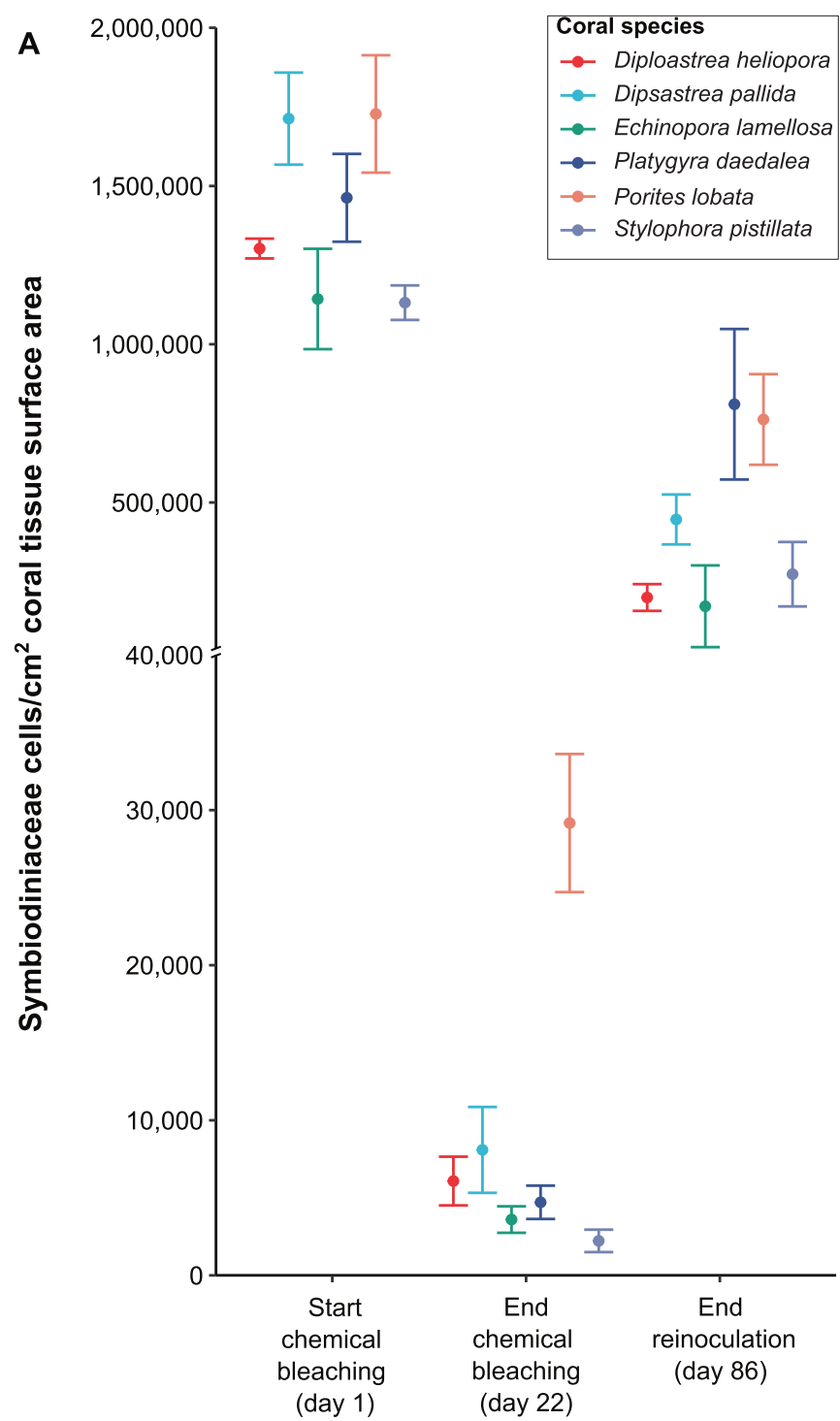

Timepoint

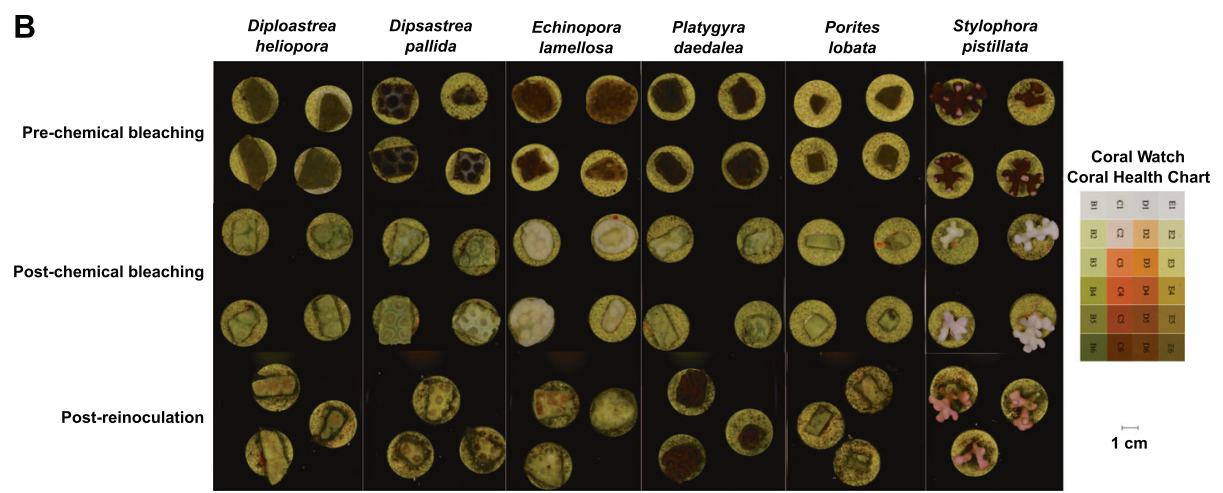

Fig. 1 Coral bleaching and repigmentation responses to chemical bleaching and reinoculation. A Symbiodiniaceae cell densities in hospite before and after chemical bleaching ( $n=4$ per species) and after reinoculation ( $n=6$ per species, except for E. lamellosa: $n=2 ;$ P.daedalea: $n=$ 5; S. pistillata: $n=5$ ). Error bars represent 1 standard error. B Images of corals before and after chemical bleaching and nine weeks after reinoculation with a cultured Cladocopium $\mathrm{C1}^{\text {acro }}$ strain in the presence of sterilised sand (treatment RiS shown here). Both $D$. pallida and D. heliopora displayed thinner tissue after reinoculation resulting in their septa standing out. No tissue necrosis was recorded in these coral species, contrarily to E. lamellosa and P. lobata fragments. The Coral Watch Coral Health Chart depicts differences in pigmentation when a coral undergoes bleaching. 


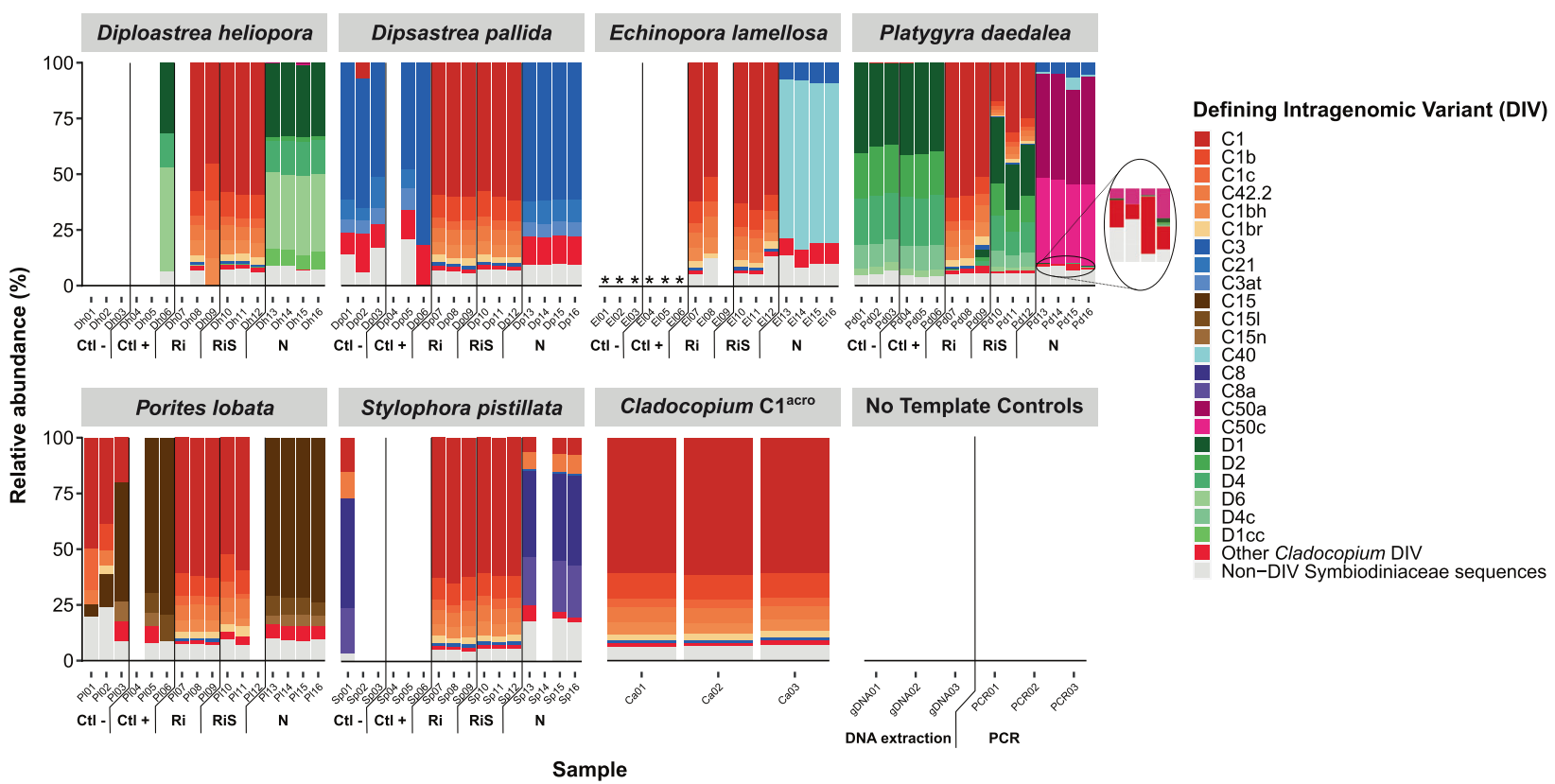

Fig. 2 Relative abundance of Symbiodiniaceae community (ITS2) profiles. Symbiodiniaceae community profiles in the six coral species before bleaching and 9 weeks after inoculation and the cultured Cladocopium $\mathrm{C}^{\text {acro }}$ strain used for reinoculation. Defining intragenomic variants (DIVs) are recurring assemblages of ITS2 sequences found at set abundances across samples. Each bar represents a sample from a coral fragment ( $n=3$ per reinoculation treatment, $n=4$ for treatment $\mathrm{N}$ ), with each individual bar representing the proportion of a DIV sequence relative to the total abundance of sequences. Empty bars correspond to samples for which no reads were obtained or which were removed for possessing only one DIV (considered as insufficient sequence depth). No-template controls from the DNA extraction and PCR amplification ( $n=3$ for each) were also sequenced and are shown. $\mathrm{Ctl}-=$ negative control treatment where corals were chemically bleached but not reinoculated with any Symbiodiniaceae. $\mathrm{Ctl}+=$ positive control treatment where corals were chemically bleached and then reinoculated with freshly isolated homologous Symbiodiniaceae. $\mathrm{RI}=$ reinoculation treatment where corals were reinoculated with a cultured Cladocopium $\mathrm{C} 1^{\text {acro }}$ strain. $\mathrm{RiS}=$ reinoculation with sand treatment where corals were reinoculated as in treatment Ri in the presence of sterilised sand. $\mathrm{N}=$ native Symbiodiniaceae community before bleaching. ${ }^{*}=$ dead corals.

cells/ml; (4) a reinoculation with sand treatment (RiS) where corals were reinoculated as in treatment $\mathrm{Ri}$ in the presence of sterilised sand (Supplementary methods 1.4).

Coral pigmentation, a phenotypic trait partly dependent on Symbiodiniaceae abundance, was scored to assess the progression of bleaching and symbiosis establishment in the corals following the methodology established by Quigley et al. [16]; see Supplementary methods 1.5 . Symbiodiniaceae cell density in hospite was quantified before and after chemical bleaching, and nine weeks after the first reinoculation to assess the extent of bleaching and recovery (Supplementary methods 1.6).

A decrease in pigmentation and a $98.3-99.8 \%$ reduction in Symbiodiniaceae density between the start and end of chemical bleaching was recorded in all six coral species (Figs. 1A, B; S1). Following reinoculation with the Cladocopium $C 1^{\text {acro }}$ strain, repigmentation was observed across all six coral species (Figs. 1B; S2). Pigmentation scores and symbiont densities were higher in treatments $\mathrm{Ri}$ and $\mathrm{RiS}$ than in treatment $\mathrm{Ctl}$-, except for $P$. daedalea which repigmented equally across all treatments (Figs. S2, S3).

Sequencing of the Internal Transcribed Spacer 2 (ITS2) region of the Symbiodiniaceae nrDNA was undertaken to characterise the symbiont communities before bleaching and nine weeks after the first reinoculation with Cladocopium $\mathrm{Cl}^{\text {acro }}$ (Table S1; Supplementary methods 1.6). The native Symbiodiniaceae communities of the six coral species were genetically distinct from the Cladocopium $\mathrm{C} 1^{\text {acro }}$ phylotype used for reinoculation (Fig. 2). In all six coral species, the native Symbiodiniaceae were replaced with the inoculum Cladocopium $\mathrm{C1}^{\text {acro }}$ in treatments $\mathrm{Ri}$ and RiS, while this Cladocopium $\mathrm{C}^{\text {acro }}$ strain was not detected in the native symbiont communities of the corals (Figs. 2, S3-S4; Table S2), demonstrating that severely bleached adult corals are able to acquire new symbionts from the environment.
The environmental acquisition of the Cladocopium $\mathrm{C} 1^{\text {acro }}$ strain by $P$. lobata and S. pistillata contributes to the growing body of evidence that vertically transmitting corals also possess the ability to acquire symbionts horizontally [17]. The presence of sand during reinoculation had a measurable effect on the composition of the Symbiodiniaceae community in $P$. daedalea only, which led to a mixed community with a Durusdinium phylotype that was initially detected in low abundance $(<1 \%)$.

Chemical bleaching is increasingly used in studies investigating cnidarian-symbiont interactions to obtain aposymbiotic hosts for reinfection with cultured Symbiodiniaceae [13, 18]. Adult Stylophora pistillata and Isopora palifera corals have been found to lose $99 \%$ of their algal symbiont densities following menthol exposure [19]. Comparable levels of Symbiodiniaceae density reduction (98.3-99.8\%) were observed for the six coral species used here. Unlike Exaiptasia diaphana [18], none of the coral species in this study were rendered completely aposymbiotic from exposure to menthol and DCMU. Severe reduction of the native Symbiodiniaceae communities, rather than complete elimination, is thus sufficient for novel symbioses to establish, though the long-term stability (>9 weeks after reinoculation) of these symbioses needs to be investigated.

Our findings provide compelling evidence that adult fragments of six coral species, spanning four families in the complex $(P$. lobata) and robust ( $D$. heliopora, S. pistillata, D. pallida, E. lamellosa, $P$. daedalea) clades, can acquire heterologous Cladocopium $\mathrm{C1}^{\text {acro }}$ symbionts from the environment. The chemical bleaching and reinoculation methodology may prove highly relevant for the study of coral-Symbiodiniaceae interactions and for the development of adult coral stock with enhanced thermal tolerance for reef restoration by inoculating them with heat-evolved Symbiodiniaceae [20]. 


\section{DATA AVAILABILITY}

Raw sequences of the ITS2 Symbiodiniaceae datasets are available in GenBank (BioSample accession: SAMN22047733 - SAMN22047828; BioProject ID: PRJNA768535).

\section{REFERENCES}

1. Falkowski PG, Dubinsky Z, Muscatine L, Porter JW. Light and the Bioenergetics of a Symbiotic Coral. BioScience 1984;34:705-9.

2. Baird $\mathrm{AH}$, Marshall PA. Mortality, growth and reproduction in scleractinian corals following bleaching on the Great Barrier Reef. Mar Ecol Prog Ser. 2002;237:133-41.

3. Pratchett MS, Heron SF, Mellin C, Cumming GS. Recurrent Mass-Bleaching and the Potential for Ecosystem Collapse on Australia's Great Barrier Reef. In: Canadell JG, Jackson RB, editors. Ecosystem Collapse and Climate Change. Ecological Studies (Analysis and Synthesis). Cham, Zug, CH:Springer International Publishing; 2021. p. 265-289.

4. Mieog JC, Olsen JL, Berkelmans R, Bleuler-Martinez SA, Willis BL, van Oppen MJH. The roles and interactions of symbiont, host and environment in defining coral fitness. PLoS ONE. 2009;4:e6364.

5. Berkelmans R, Van, Oppen MJH. The role of zooxanthellae in the thermal tolerance of corals: a "nugget of hope" for coral reefs in an era of climate change. Proc R Soc B. 2006:273:2305-12.

6. Silverstein RN, Cunning R, Baker AC, Tenacious D. Symbiodiniumin clade D remain in reef corals at both high and low temperature extremes despite impairment. J Exp Biol. 2017;220:1192-6.

7. Pochon X, LaJeunesse TC. Miliolidium n. gen, a New Symbiodiniacean Genus Whose Members Associate with Soritid Foraminifera or Are Free-Living. J Eukaryot Microbiol. 2021;68:e12856.

8. Silverstein RN, Correa AM, Baker AC. Specificity is rarely absolute in coral-algal symbiosis: implications for coral response to climate change. Proc R Soc B. 2012;279:2609-18.

9. Ziegler M, Eguíluz VM, Duarte CM, Voolstra CR. Rare symbionts may contribute to the resilience of coral-algal assemblages. ISME J. 2018;12:161-72.

10. Baker AC, Starger CJ, McClanahan TR, Glynn PW. Corals' adaptive response to climate change: shifting to new algal symbionts may safeguard devastated reefs from extinction. Nature 2004;430:741.

11. Boulotte NM, Dalton SJ, Carroll AG, Harrison PL, Putnam HM, Peplow LM, et al. Exploring the Symbiodinium rare biosphere provides evidence for symbiont switching in reef-building corals. ISMEJ. 2016;10:2693-701.

12. Rouzé $H$, Lecellier G, Pochon X, Torda G, Berteaux-Lecellier V. Unique quantitative Symbiodiniaceae signature of coral colonies revealed through spatio-temporal survey in Moorea. Sci Rep. 2019;9:7921.

13. Herrera M, Klein SG, Schmidt-Roach S, Campana S, Cziesielski MJ, Chen JE, et al. Unfamiliar partnerships limit cnidarian holobiont acclimation to warming. Glob Change Biol. 2020;26:5539-55535.

14. Lewis CL, Coffroth MA. The Acquisition of Exogenous Algal Symbionts by an Octocoral After Bleaching. Science 2004;304:1490-2.

15. Coffroth MA, Poland DM, Petrou EL, Brazeau DA, Holmberg JC. Environmental symbiont acquisition may not be the solution to warming seas for reef-building corals. PLoS ONE. 2010;5:e13258.

16. Quigley KM, Willis BL, Kenkel CD. Transgenerational inheritance of shuffled symbiont communities in the coral Montipora digitata. Sci Rep. 2019;9:13328.

17. Byler KA, Carmi-Veal M, Fine M, Goulet TL. Multiple Symbiont Acquisition Strategies as an Adaptive Mechanism in the Coral Stylophora pistillata. PLoS ONE. 2013;8:e59596.

18. Matthews JL, Sproles AE, Oakley CA, Grossman AR, Weis VM, Davy SK. Mentholinduced bleaching rapidly and effectively provides experimental aposymbiotic sea anemones (Aiptasia sp.) for symbiosis investigations. J Exp Biol. 2016;219:306-10.

19. Wang JT, Chen YY, Tew KS, Meng PJ, Chen CA. Physiological and Biochemical Performances of Menthol-Induced Aposymbiotic Corals. PLoS ONE. 2012;7:e46406.

20. Buerger P, Alvarez-Roa C, Coppin CW, Pearce SL, Chakravarti L, Oakeshott GJ, et al. Heat-evolved microalgal symbionts increase coral bleaching tolerance. Sci Adv. 2020;6:eaba2498.

\section{ACKNOWLEDGEMENTS}

We thank D. Baker, S. Mcllroy and G. Puntin for fruitful discussion on the menthol bleaching method. This research was supported by the Australian Research Council Laureate Fellowship to MJHvO (FL180100036), the Paul G Allen Family Foundation, the Reef Restoration and Adaptation Program, which is funded by the partnership between the Australian Governments Reef Trust and the Great Barrier Reef Foundation, and the University of Melbourne (funding received from the North Australian Animal Trust scholarship to HJS).

\section{AUTHOR CONTRIBUTIONS}

All authors participated in the conception and design of the study. HS carried out the experiment and sample processing. HS, WC and PB performed the data analysis and figure drawing. $\mathrm{HS}, \mathrm{WC}, \mathrm{PB}$ and $\mathrm{MvO}$ provided critical biological interpretations of the data. HS wrote the paper. WC, $\mathrm{PB}, \mathrm{CH}$ and $\mathrm{MvO}$ supervised this work and edited the paper.

\section{FUNDING}

Open Access funding enabled and organized by CAUL and its Member Institutions.

\section{COMPETING INTERESTS}

This research was supported by the Australian Research Council Laureate Fellowship to MJHvO (FL180100036), the Paul G. Allen Family Foundation, the Reef Restoration and Adaptation Program, which is funded by the partnership between the Australian Governments Reef Trust and the Great Barrier Reef Foundation, and the University of Melbourne (funding received from the North Australian Animal Trust scholarship to HJS). The authors declare no competing interests.

\section{ADDITIONAL INFORMATION}

Supplementary information The online version contains supplementary material available at https://doi.org/10.1038/s41396-022-01203-0.

Correspondence and requests for materials should be addressed to Hugo J. Scharfenstein.

Reprints and permission information is available at http://www.nature.com/ reprints

Publisher's note Springer Nature remains neutral with regard to jurisdictional claims in published maps and institutional affiliations.

Open Access This article is licensed under a Creative Commons Attribution 4.0 International License, which permits use, sharing, adaptation, distribution and reproduction in any medium or format, as long as you give appropriate credit to the original author(s) and the source, provide a link to the Creative Commons license, and indicate if changes were made. The images or other third party material in this article are included in the article's Creative Commons license, unless indicated otherwise in a credit line to the material. If material is not included in the article's Creative Commons license and your intended use is not permitted by statutory regulation or exceeds the permitted use, you will need to obtain permission directly from the copyright holder. To view a copy of this license, visit http://creativecommons. org/licenses/by/4.0/.

(c) The Author(s) 2022 